\title{
ACTIVE MANIPULATION OF FIELDS MODELED BY THE HELMHOLTZ EQUATION
}

\author{
DANIEL ONOFREI
}

\begin{abstract}
In this paper we extend the results proposed in [36] and study the problem of active control in the context of a scalar Helmholtz equation. Given a source region $D_{a}$ and $\left\{v_{0}, v_{1}, \ldots, v_{n}\right\}$, a set of solutions of the homogeneous scalar Helmholtz equation in $n$ mutually disjoint "control" regions $\left\{D_{0}, D_{1}, \ldots, D_{n}\right\}$ of $\mathbb{R}^{2}$ or $\mathbb{R}^{3}$, respectively, the main objective of this paper is to characterize the necessary boundary data on $\partial D_{a}$ so that the solution to the corresponding exterior scalar Helmholtz problem will closely approximate $v_{i}$ in $D_{i}$, respectively, for each $i \in\{0, \ldots, n\}$. Building up on the previous ideas presented in [36] we show the existence of a class of solutions to the problem, provide numerical support of the results in $2 \mathrm{D}$ and $3 \mathrm{D}$ and discuss the existence of a minimal energy solution and its stability.
\end{abstract}

1. Introduction. During recent years, there has been a growing interest in the development of feasible strategies for the control of acoustic and electromagnetic fields with one possible application being the construction of robust schemes for sonar or radar cloaking.

One main approach controls fields in the regions of interest by changing the material properties of the medium in certain surrounding regions $[4,5,8,13,14,15,39]$ (and the references therein). Several alternative techniques are proposed in the literature (other than transformation optics strategies) such as: plasmonic designs (see [2] and the references therein), strategies based on anomalous resonance phenomena (see $[\mathbf{3 1}, \mathbf{3 2}, \mathbf{3 3}]$ ), conformal mapping techniques (see $[\mathbf{2 4}, \mathbf{2 5}]$ ), and complementary media strategies (see [23]).

In this paper, we will study an approximate control problem for the exterior scalar Helmholtz equation, i.e., we will characterize the boundary control functions on the source $\partial D_{a}$ so that we achieve

Keywords and phrases. Active manipulation, Helmholtz equation, layer potentials, integral equation, active exterior cloaking.

The AFOSR supported this work under the 2013 YIP Award FA9550-13-1-0078.

Received by the editors on May 23, 2014. 
desired control effects in the regions $\left\{D_{1}, D_{2}, \ldots, D_{n}\right\}$ of $\mathbb{R}^{2}$ or $\mathbb{R}^{3}$. The method is novel in the sense that, instead of using microstructures, now exterior active sources, modeled with the help of the above boundary controls (which can represent velocity potential, pressure or currents), are employed for the desired control effects.

In the applied community, active designs for the manipulation of fields appear to have occurred initially in the context of low-frequency acoustics (or active noise cancellation). Especially notable are the pioneer works of Leug [26] (feed-forward control of sound) and Olson and May [35] (feedback control of sound). The reviews $[\mathbf{1 1}, \mathbf{1 2}, \mathbf{2 7}$, $\mathbf{2 8}, \mathbf{3 8}, \mathbf{4 0}$ ], provide detailed accounts of past and recent developments in acoustic active control.

In the context of cloaking, the interior strategy proposed in [30] employs a continuous active layer on the boundary of the control region while the exterior scheme discussed in $[\mathbf{1 7}, \mathbf{1 6}, \mathbf{1 8}, \mathbf{1 9}$ ] (see also [43]) uses a discrete number of active sources located in the exterior of the control region to manipulate the fields. The active exterior strategy for $2 \mathrm{D}$ quasistatics cloaking was introduced in [16] and, based on $a$ priori information about the incoming field the authors constructively described how one can create an almost zero field control region with very small effect in the far field. The proposed strategy did not work for control regions close to the active source, it "cloaked" large objects only when they were far enough from the source region (see [17]) and was not adaptable for three space dimensions. The finite frequency case was studied in the last section of [16] and in [19] (see also [17] for a recent review) where three (or four in 3D) active sources were needed to create a zero field region in the interior of their convex hull while creating a very small scattering effect in the far field. The broadband character of the proposed scheme was numerically observed in [18]. Experimental designs and testing of active cloaking schemes in various regimes are reported in $[\mathbf{9}, \mathbf{2 9}, \mathbf{4 1}, \mathbf{4 2}]$.

In a recent development in [36], a general analytical approach based on the theory of boundary layer potentials is proposed for the active control problem in the quasi-static regime. In this spirit, this paper extends the results presented in [36] to the case of scalar Helmholtz equation.

The paper is organized as follows. In Section 2, we state the main 
results of the paper Theorem 2.1 and Corollary 2.2. Then, in Section 3 we present the proofs for the results, characterize a class of solutions for Theorem 2.1 and Corollary 2.2 and discuss the corresponding minimal energy solution. In Section 4, we show several numerical simulations to support our existence results. In Section 5, we provide a brief discussion about the stability of the minimal energy solution. We conclude the paper with Section 6 where we highlight the potential impact of the results and reiterate some of the ongoing challenges we face.

2. Statement of main results. In this section, we will state the general results of the paper, Theorem 2.1 with Corollary 2.2, and then, as an important example, in Remark 2.3 we will reformulate it in the particular context of cloaking.

First, we proceed with the description of the geometry and functional framework. Let us consider the active source region, i.e., a smooth domain $D_{a} \subset \mathbb{R}^{d}$, (where $d=\overline{2,3}$ ) and let $D_{a^{\prime}}$ be such that $D_{a^{\prime}} \subset \subset$ $D_{a}$ where here and in the remainder of the paper " $\subset \subset$ " denotes a compact embedding and, by a domain, we understand an open simplyconnected subset of $\mathbb{R}^{d}$. Let $n \in \mathbb{N}$, consider $n+1$ smooth mutually disjoint domains (control regions) $\left\{D_{0}, D_{1}, \ldots, D_{n}\right\}$ in $\mathbb{R}^{d}$, and let the sets $\left\{W_{i}\right\}_{i=0}^{n}$ be smooth mutually disjoint sub-domains of $\mathbb{R}^{d}$ with $D_{i} \subset \subset W_{i}$ for $i=\overline{0, n}$. Assume further that the antenna and the regions of control are well separated,

$$
\bar{W}_{i} \cap \bar{D}_{a}=\emptyset, \quad \text { for } i=\overline{0, n},
$$

and consider the following geometrical settings:

I. $W_{i}$ is bounded for $i=\overline{0, n}$

II. $W_{i}$ is bounded for $i=\overline{1, n}$ and $W_{0}=\mathbb{R}^{d} \backslash \bar{B}$ for some

$$
\text { smooth bounded domain } B \text { with }\left\{D_{a} \cup \bigcup_{i=\overline{1, n}} W_{i}\right\} \subset \subset B \text {. }
$$

Consider also a set of $n+1$ functions $\left\{v_{i}\right\}_{i=0}^{n}$ such that:

$$
v_{i} \in L^{2}\left(\partial W_{i}\right), \quad \text { for each } i=\overline{0, n} .
$$


The main mathematical result of the paper is stated below in Theorem 2.1 and shows the theoretical possibility of characterizing needed input data on the boundary of the active source $D_{a}$ so that, given desired fields, $\left\{v_{i}\right\}_{i}$ are well approximated in prescribed bounded or unbounded mutually disjoint regions of space, $\left\{W_{i}\right\}_{i}$, respectively. We have

Theorem 2.1. Let $0<\delta \ll 1$ be fixed, and consider $\{W\}_{i}$ as above with either of the cases I or II possible. In addition, assume

i) $-k^{2}$ is not a Neumann eigenvalue for the Laplacian in $D_{a^{\prime}}$.

ii) For each $i=\overline{0, n}$, if $W_{i}$ is bounded, $-k^{2}$ is not a Laplace

Dirichlet eigenvalue in $W_{i}$.

There exists an infinite class of functions $v_{b} \in C\left(\partial D_{a}\right)$ such that there exists $u \in H^{1}\left(\mathbb{R}^{d} \backslash \bar{D}_{a}\right)$, satisfying

$$
\left\{\begin{array}{l}
\Delta u+k^{2} u=0 \text { in } \mathbb{R}^{d} \backslash \bar{D}_{a}, \\
u=v_{b} \text { on } \partial D_{a}, \\
\left(\frac{x}{|x|}, \nabla u(x)\right)-i k u(x)=o\left(\frac{1}{|x|^{d-1 / 2}}\right), \\
\text { as }|x| \rightarrow \infty \text { uniformly for all } \frac{x}{|x|}
\end{array}\right.
$$

and

$$
\left\|u-v_{i}\right\|_{L^{2}\left(\partial W_{i}\right)} \leq \delta
$$

Next, using classical estimates for the boundary value problems associated to the Helmholtz equation, one can obtain the following important corollary of Theorem 2.1:

Corollary 2.2. Assume that, for $i=\overline{0, n}$, the functions $v_{i}$ introduced in Theorem 2.1 are solutions of the homogeneous scalar Helmholtz equation in $W_{i}$, respectively. Assume also that, if (2.3) holds, then we also have

$$
-k^{2} \text { is not a Neumann eigenvalue for the Laplacian in B. }
$$


Then, in the setting of Theorem 2.1, for each $v_{b} \in C\left(\partial D_{a}\right)$, u, the corresponding solutions of (2.7) satisfy

$$
\left\|u-v_{i}\right\|_{C^{2}\left(D_{i}\right)} \leq C_{i} \delta, \quad \text { for } i=\overline{0, n}
$$

where, for each $i=\overline{0, n}, C_{i}=C_{i}\left(\operatorname{dist}\left(\partial D_{i}, \partial W_{i}\right), k, D_{a}\right)$.

Remark 2.3. The geometrical setting described in (2.3) corresponds to the active cloaking problem. Indeed, let $v_{0}=0$ and $v_{i}=-u_{\text {int }}$ for $i=\overline{1, n}$, where $u_{i n t}$ is a solution of the Helmholtz equation in $B$. Then, in this new context, the results presented in Corollary 2.2 correspond to the problem of acoustic or electromagnetic cloaking, where the active source cancels an incoming interrogating field $u_{i n t}$ in different control regions while maintaining a very low profile to a far field observer.

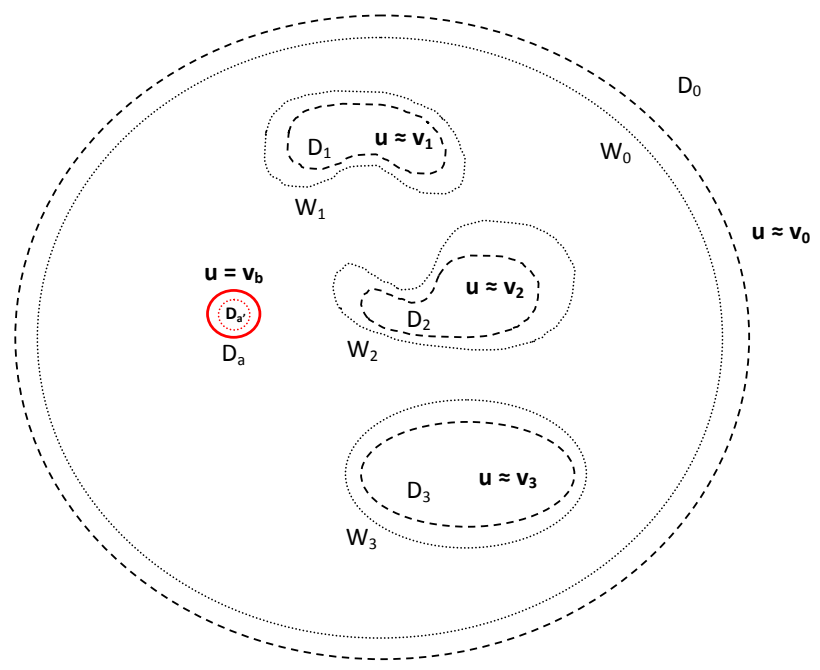

FIGURE 1. 2D sketch of geometrical setting described at (2.3) for $n=3$.

\section{Proofs.}

3.1. The case $n=1$. In this section, we will consider the case $n=1$ and, in subsection 3.2 , we will show how the proofs can then 
be immediately extended to the general case. Note that, in the case $n=1$, the two possible geometrical configurations presented at $(2.2)$, (2.3) become:

I. $W_{0}, W_{1}$ are bounded,

II. $W_{1}$ is bounded and $W_{0}=\mathbb{R}^{d} \backslash \bar{B}$ for some smooth bounded domain $B$ with $W_{1} \cup D_{a} \subset \subset B$,

and the functions to be approximated are, $v_{1} \in L^{2}\left(\partial W_{1}\right)$ and $v_{0} \in$ $L^{2}\left(\partial W_{0}\right)$ as described in (2.4) for $n=1$.

It is well known that, (see [7]), given $v_{b} \in C\left(\partial D_{a}\right)$, problem (2.7) has a unique solution in $H^{1}\left(\mathbb{R}^{d} \backslash \bar{D}_{a}\right)$. Hence, the question is: what are the boundary functions $v_{b} \in C\left(\partial D_{a}\right)$ such that (2.8) holds? The proof will be based on the ideas presented in [36] and, in order to avoid redundant arguments, where the proof is very similar to a result in [36], we will only state the result.

As in [36], we introduce the following space $\Xi$,

$$
\Xi \equiv L^{2}\left(\partial W_{1}\right) \times L^{2}\left(\partial W_{0}\right) .
$$

Then $\Xi$ is a Hilbert space with respect to the scalar product given by

$$
(\varphi, \psi)_{\Xi}=\int_{\partial W_{1}} \varphi_{1}(\mathbf{y}) \bar{\psi}_{1}(\mathbf{y}) d s_{\mathbf{y}}+\int_{\partial W_{0}} \varphi_{2}(\mathbf{y}) \bar{\psi}_{2}(\mathbf{y}) d s_{\mathbf{y}},
$$

for all $\varphi \equiv\left(\varphi_{1}, \varphi_{2}\right)$ and $\psi=\left(\psi_{1}, \psi_{2}\right)$ in $\Xi$, where $\left(\bar{c}^{-}\right)$above, denotes the complex conjugate. Let $k$ satisfy (2.5) and (2.6). Consider the following integral operator, $K: L^{2}\left(\partial D_{a^{\prime}}\right) \rightarrow \Xi$, defined as

$$
K u(\mathbf{x}, \mathbf{z})=\left(K_{1} u(\mathbf{x}), K_{2} u(\mathbf{z})\right),
$$

for any $u \in L^{2}\left(\partial D_{a^{\prime}}\right)$, where

$$
\begin{aligned}
& K_{1} u(\mathbf{x})=\int_{\partial D_{a^{\prime}}} u(\mathbf{y}) \frac{\partial \Phi(\mathbf{x}, \mathbf{y})}{\partial \boldsymbol{\nu}_{\mathbf{y}}} d s_{\mathbf{y}}, \quad \text { for } \mathbf{x} \in \partial W_{1}, \\
& K_{2} u(\mathbf{z})=\int_{\partial D_{a^{\prime}}} u(\mathbf{y}) \frac{\partial \Phi(\mathbf{z}, \mathbf{y})}{\partial \boldsymbol{\nu}_{\mathbf{y}}} d s_{\mathbf{y}}, \quad \text { for } \mathbf{z} \in \partial W_{0},
\end{aligned}
$$

with $\boldsymbol{\nu}_{\mathbf{y}}$ denoting the unit normal exterior to $\partial D_{a^{\prime}}$ and where $\Phi(\mathbf{x}, \mathbf{y})$ represents the fundamental solution of the relevant Helmholtz operator, 
i.e.,

$$
\Phi(\mathbf{x}, \mathbf{y})=\left\{\begin{array}{l}
\frac{e^{i k|\mathbf{x}-\mathbf{y}|}}{4 \pi|\mathbf{x}-\mathbf{y}|}, \quad \text { for } d=3 \\
\frac{i}{4} H_{0}^{(1)}(k|\mathbf{x}-\mathbf{y}|), \quad \text { for } d=2
\end{array}\right.
$$

In what follows, some steps will be similar as in [36], but for clarity of the exposition we prefer to included them here. Let us consider the integral operator, $K: L^{2}\left(\partial D_{a^{\prime}}\right) \rightarrow \Xi$, defined at (3.5) and (3.6), and introduce its adjoint, i.e., the operator $K^{*}: \Xi \rightarrow L^{2}\left(\partial D_{a^{\prime}}\right)$ defined through the relation

$$
(K v, u)_{\Xi}=\left(v, K^{*} u\right)_{L^{2}\left(\partial D_{a^{\prime}}\right)}, \quad \text { for all } u \in \Xi, v \in L^{2}\left(\partial D_{a^{\prime}}\right)
$$

where $(\cdot, \cdot) \Xi$ is the scalar product on $\Xi$ defined in 3.4 and $(\cdot, \cdot)_{L^{2}\left(\partial D_{a^{\prime}}\right)}$ denotes the usual scalar product in $L^{2}\left(\partial D_{a^{\prime}}\right)$ defined as a vectorial space over the complex field. We check, by a simple change of variables and algebraic manipulations, that the adjoint operator $K^{*}$ is given by

$$
K^{*} u(\mathbf{x})=\int_{\partial W_{1}} u_{1}(\mathbf{y}) \frac{\partial \bar{\Phi}(\mathbf{x}, \mathbf{y})}{\partial \boldsymbol{\nu}_{\mathbf{x}}} d s_{\mathbf{y}}+\int_{\partial W_{0}} u_{2}(\mathbf{y}) \frac{\partial \bar{\Phi}(\mathbf{x}, \mathbf{y})}{\partial \boldsymbol{\nu}_{\mathbf{x}}} d s_{\mathbf{y}}
$$

for any $u=\left(u_{1}, u_{2}\right) \in \Xi$ and $\mathbf{x} \in \partial D_{a^{\prime}}$. From classical potential theory [21] (see also [7]) we have that the linear operators $K$ and $K^{*}$ defined above are compact. Furthermore, let us denote by $\operatorname{Ker}\left(K^{*}\right)$ the kernel (i.e., null space) of $K^{*}$. Then, as in [36], we obtain the following result.

Lemma 3.1. If $\psi=\left(\psi_{1}, \psi_{2}\right) \in \operatorname{Ker}\left(K^{*}\right)$ then $\psi \equiv(0,0)$ in $\Xi$.

Proof. Let $\psi=\left(\psi_{1}, \psi_{2}\right) \in \operatorname{Ker}\left(K^{*}\right)$, and define

$$
\begin{aligned}
w(\mathbf{x})= & \int_{\partial W_{1}} \bar{\psi}_{1}(\mathbf{y}) \Phi(\mathbf{x}, \mathbf{y}) d s_{\mathbf{y}} \\
& +\int_{\partial W_{0}} \bar{\psi}_{2}(\mathbf{y}) \Phi(\mathbf{x}, \mathbf{y}) d s_{\mathbf{y}} \quad \text { for } \mathbf{x} \in \mathbb{R}^{d}
\end{aligned}
$$

where the integrals exist as improper integrals for $\mathbf{x} \in \partial W_{1} \cup \partial W_{0}$. 
From $K^{*} \psi=0$ and (3.9) we have that $w$ satisfies

$$
\begin{cases}\Delta w+k^{2} w=0, & \text { in } D_{a^{\prime}} \\ \frac{\partial w}{\partial \boldsymbol{\nu}_{\mathbf{x}}}=0, & \text { on } \partial D_{a^{\prime}} .\end{cases}
$$

From (2.5), we conclude that

$$
w=0 \text { in } D_{a^{\prime}} .
$$

Then, because by definition $w$ is a solution of Helmholtz equation in $\mathbb{R}^{d} \backslash\left\{\bar{W}_{0} \cup \bar{W}_{1}\right\}$, by analytic continuation we conclude that

$$
w=0 \text { in } \mathbb{R}^{d} \backslash\left\{\bar{W}_{0} \cup \bar{W}_{1}\right\} .
$$

where we used the fact that the jump relations for the layer potentials are valid for domains with multiple components. Next, we will make use of the classical interior and exterior jump conditions for the layer potentials with $L^{2}$ densities and their adjoints (see [6] and the references therein). Thus, from the continuity of the single layer potential, we have

$$
w=0 \quad \text { on } \partial W_{1} \cup \partial W_{0} .
$$

Hypotheses (2.6) and (3.14) imply

$$
w=0 \text { in } \bar{W}_{1} .
$$

By using (3.13), (3.15), and the interior and exterior jump relations for the adjoint of the double layer potential on $\partial W_{1}$, we obtain that

$$
\psi_{1}=0 \quad \text { on } \partial W_{1} .
$$

Equation (3.16) implies

$$
w(\mathbf{x})=\int_{\partial W_{0}} \bar{\psi}_{2}(\mathbf{y}) \Phi(\mathbf{x}, \mathbf{y}) d s_{\mathbf{y}}, \quad \text { for } \mathbf{x} \in \mathbb{R}^{d} .
$$

Note also that (3.13), (3.14), and (3.15) imply that

$$
w=0 \text { in } \mathbb{R}^{d} \backslash W_{0} .
$$

Now, if (3.1) is true, then we may use the same ideas as above for $w$ given by (3.17) to show that

$$
\psi_{2}=0 \quad \text { on } \partial W_{0} .
$$


If, on the other hand, (3.1) is false, then $W_{0}$ is an exterior domain and, unless we impose further restriction on $k$ (to insure uniqueness of the Dirichlet exterior problem) we cannot use the same tools as we did above.

Thus, we need to proceed as follows. From the interior jump condition for the adjoint of the double layer potential over $\partial W_{0}=\partial B$, together with (3.18), we have that

$$
\frac{\partial w}{\partial \boldsymbol{\nu}_{\mathbf{x}}}=-\frac{1}{2} \bar{\psi}_{2} \quad \text { almost everywhere on } \partial B \text {. }
$$

Since $w$ is a solution of the Helmholtz equation in $\mathbb{R}^{d} \backslash W_{0}=\bar{B}$, by using the Green representation theorem for $\mathbf{x} \in \mathbb{R}^{d} \backslash \bar{B}$, we obtain

$$
\begin{aligned}
0 & =\int_{\partial B} \frac{\partial w}{\partial \nu_{\mathbf{y}}}(\mathbf{y}) \Phi(\mathbf{x}, \mathbf{y}) d s_{\mathbf{y}}-\int_{\partial B} w(\mathbf{y}) \frac{\partial \Phi(\mathbf{x}, \mathbf{y})}{\partial \nu_{\mathbf{y}}}(\mathbf{y}) d s_{\mathbf{y}} \\
& =-\frac{1}{2} \int_{\partial B} \bar{\psi}_{2}(\mathbf{y}) \Phi(\mathbf{x}, \mathbf{y}) d s_{\mathbf{y}} \\
(3.21) & =-\frac{1}{2} w(\mathbf{x}),
\end{aligned}
$$

where we used (3.14), (3.17) and (3.20) in the equalities above. Thus, from (3.21) and the jump conditions, the adjoint of the second layer potential over $\partial W_{0}=\partial B$, we obtain $\psi_{2}=0$ on $\partial W_{0}$.

Next, as a consequence of Lemma 3.1, we obtain:

Proposition 3.1. The operator $K$ is compact and has a dense range.

Proof. As in [36], consider

$$
U \equiv K\left(C\left(\partial D_{a^{\prime}}\right)\right) .
$$

Then $U$ is a subspace of $\Xi$ and, moreover, following similar arguments as in [36], Lemma 3.1 implies that the set $U \subset \Xi$ is dense in $\Xi$, and thus the statement of Proposition 3.1 is proved.

Proof of Theorem 2.1. The statement of Theorem 2.1 now follows immediately. Indeed, the result of Proposition 3.1 implies that there 
exists $\left\{w_{j}\right\}_{j} \subset C\left(\partial D_{a^{\prime}}\right)$, such that

$$
K w_{j} \longrightarrow\left(v_{1}, v_{0}\right), \quad \text { in } \Xi .
$$

Let us consider the double layer potential $\mathcal{D}$ with density $\varphi \in C\left(\partial D_{a^{\prime}}\right)$, defined as

$$
\mathcal{D} \varphi(\mathbf{x})=\int_{\partial D_{a^{\prime}}} \varphi(\mathbf{y}) \frac{\partial \Phi(\mathbf{x}, \mathbf{y})}{\partial \nu_{\mathbf{y}}} d s_{\mathbf{y}}, \quad \text { for } \mathbf{x} \in \mathbb{R}^{d} \backslash \bar{D}_{a^{\prime}} .
$$

Then, from (3.22), we have that, for any $\delta \ll 1$, there exists an infinity of indices $j \in \mathbb{N}$ such that, for $v_{b}=\mathcal{D} w_{j}$, on $\partial D_{a}$, the function $u(\mathbf{z})=\int_{\partial D_{a^{\prime}}} w_{j}(\mathbf{y}) \partial \Phi(\mathbf{z}, \mathbf{y}) / \partial \nu_{\mathbf{y}} d s_{\mathbf{y}}$, for $\mathbf{z} \in \mathbb{R}^{d} \backslash \bar{D}_{a}$ will be the unique solution of (2.7) and satisfy (2.8).

Remark 3.1. We make the observation that the fictitious domain $D_{a^{\prime}}$ with $D_{a^{\prime}} \subset \subset D_{a}$ helped us obtain a very smooth datum $v_{b}$ on the actual physical source boundary $D_{a}$ for the desired controls, and this fact will be very important when we will make use of these results for the similar control problem for Maxwell equations in free space or waveguides $([\mathbf{1}, \mathbf{3 7}])$ through the Debye potentials representations.

Remark 3.2. Consider $v_{1} \in L^{2}\left(\partial W_{1}\right)$ and $v_{0} \in L^{2}\left(\partial W_{0}\right)$ as in (2.4) for $n=1$. One possible way to obtain the functions $w_{j}$ in (3.22) is through the Tikhonov regularization $[\mathbf{3}, \mathbf{1 0}]$. Indeed, for $\alpha_{j} \rightarrow 0$ (i.e., Tikhonov regularizers) the functions $w_{j} \in C\left(\partial D_{a^{\prime}}\right)$ defined by

$$
w_{j}=\left(\alpha_{j} I+K^{*} K\right)^{-1} K^{*} v, \quad \text { with } v=\left(v_{1}, v_{0}\right),
$$

have the property that, for any $\delta<<1$, there exists an $N_{\delta} \in \mathbb{N}$ such that, for any $j>N_{\delta}$, the functions $u_{j}$ given by

$$
u_{j}(\mathbf{x})=\int_{\partial D_{a^{\prime}}} w_{j}(\mathbf{y}) \frac{\partial \Phi(\mathbf{x}, \mathbf{y})}{\partial \nu_{\mathbf{y}}} d s_{\mathbf{y}}, \quad \text { for } \mathbf{x} \in \mathbb{R}^{d} \backslash \bar{D}_{a^{\prime}}
$$

satisfy

$$
\left\{\begin{array}{l}
\Delta u_{j}+k^{2} u_{j}=0, \text { in } \mathbb{R}^{d} \backslash \bar{D}_{a} \\
u_{j} \in C\left(\partial D_{a}\right) \\
\left\|u_{j}-v_{0}\right\|_{L^{2}\left(\partial W_{0}\right)} \leq \delta \\
\left\|u_{j}-v_{1}\right\|_{L^{2}\left(\partial W_{1}\right)} \leq \delta .
\end{array}\right.
$$


Proof of Corollary 2.2. Recall that we are in the case $n=1$, i.e., (3.1) or (3.2) hold and $v_{0}, v_{1}$ are solutions of the Helmholtz equation in $W_{0}$ and $W_{1}$, respectively. The next lemma presents two useful regularity results.

Lemma 3.2. Let $V$ be a smooth bounded domain in $\mathbb{R}^{d}$, and consider $U \subset \subset V$ a smooth sub-domain. Let $k \in \mathbb{R}$ be such that

(3.26) i) $-k^{2}$ is not a Dirichlet eigenvalue for the Laplacian in $V$

(3.27) ii) $-k^{2}$ is not a Neumann eigenvalue for the Laplacian in $U$.

Consider $f \in C(\partial V)$ and $g \in C(\partial U)$. Define $z_{i} \in C^{2}(V) \cap C(\bar{V})$ to be the solution of the following interior Dirichlet problem

$$
\left\{\begin{array}{l}
\Delta z_{i}+k^{2} z_{i}=0 \text { in } V \\
z_{i}=f \text { on } \partial V .
\end{array}\right.
$$

and $z_{e} \in C^{2}\left(\mathbb{R}^{d} \backslash \bar{U}\right) \cap C\left(\mathbb{R}^{d} \backslash U\right)$ as the solution of the following exterior problem,

$$
\left\{\begin{array}{l}
\Delta z_{e}+k^{2} z_{e}=0 \text { in } \mathbb{R}^{d} \backslash \bar{U} \\
z_{e}=g \text { on } \partial U, \\
\left(x /|x|, \nabla z_{e}(x)\right)-i k z_{e}(x)=o\left(1 /|x|^{d-1 / 2}\right), \\
\text { as }|x| \rightarrow \infty, \text { uniformly for all } x /|x| .
\end{array}\right.
$$

Then, we have

$$
\begin{aligned}
& \left\|z_{i}\right\|_{C^{2}(U)} \leq C\|f\|_{L^{2}(\partial V)}, \\
& \left\|z_{e}\right\|_{C^{2}\left(\mathbb{R}^{d} \backslash \bar{V}\right)} \leq C\|g\|_{L^{2}(\partial U)},
\end{aligned}
$$

where $C=C(k, U, V)$.

Proof. We first point out that the result can be obtained by adapting the proof in [7, subection 3.4]. Another way to prove (3.28) and (3.29) is by adapting the ideas in [22, subsection 6.5]. We will first sketch the proof for the interior estimate (3.28). Let $A$ denote the double layer potential with $L^{2}$ density on $\partial V$.

As in [22, subsection 6.5], by using the Fredholm alternative in different dual systems together with (3.26), we can show that $\operatorname{Ker}\left(\frac{1}{2} I-\right.$ 
$A)=0$ in $L^{2}(\partial V)$. From this and the compactness property of $A$ (which results from the Lax theorem, see [7, Theorem 4.11]) we have that $\frac{1}{2} I-A$ has a bounded inverse in $L^{2}(\partial V)$. From (3.26) and expressing the solution $z_{i}$ as a double layer potential (see [7]), we obtain

$$
z_{i}(\mathbf{z})=\int_{\partial V}\left(\frac{1}{2} I-A\right)^{-1} f(\mathbf{y}) \frac{\partial \Phi(\mathbf{z}, \mathbf{y})}{\partial \nu_{\mathbf{y}}} d s_{\mathbf{y}} \quad \text { for } \mathbf{z} \in V .
$$

Using the analyticity of $z_{i}$ in $U$, the fact that $\operatorname{dist}(U, \partial V)>0$, Cauchy inequality and the boundedness of $\left(\frac{1}{2} I-A\right)^{-1}$, we obtain (3.30). Finally, one can see that, using (3.27) and with obvious modifications of the above arguments, one can obtain the proof of (3.31).

Following similar arguments as in [36], from Proposition 3.1, Lemma 3.2 applied in our context, and the fact that, by definition, for any $\varphi \in C\left(\partial D_{a^{\prime}}\right), K_{1} \varphi$ and $K_{2} \varphi$ are restrictions of $\mathcal{D} \varphi$ on $\partial W_{1}$ and $\partial W_{0}$, respectively, we obtain that the sequence $\left\{w_{j}\right\} \subset C\left(\partial D_{a^{\prime}}\right)$ introduced at (3.22) satisfies

$$
\begin{gathered}
\left\|\mathcal{D} w_{j}-v_{1}\right\|_{C^{2}\left(D_{1}\right)} \leq C\left\|K_{1} w_{j}-v_{1}\right\|_{L^{2}\left(\partial W_{1}\right)} \rightarrow 0, \\
\left\|\mathcal{D} w_{j}-v_{0}\right\|_{C^{2}\left(D_{0}\right)} \leq C\left\|K_{2} w_{j}-v_{0}\right\|_{L^{2}\left(\partial W_{0}\right)} \rightarrow 0,
\end{gathered}
$$

for some constant $C=C\left(k, D_{1}, D_{0}, W_{0}, W_{1}\right)$. Therefore, the statement of Corollary 2.2 follows.

The next remark is a simple consequence of classical theory and states that, for any desired level of accuracy $\delta$, there exists a unique function $w_{0} \in L^{2}\left(\partial D_{a^{\prime}}\right)$ with minimal energy norm, i.e., with a minimal $L^{2}\left(\partial D_{a^{\prime}}\right)$ norm such that $\|K w-v\|_{\Xi} \leq \delta$. Indeed, Proposition 3.1 together with the classical theory of minimum norm solutions based on the Tikhonov regularization implies (see [22]):

Remark 3.3. For $0<\delta \ll 1$ and $v \in \Xi$, there exists a unique $w_{0} \in L^{2}\left(\partial D_{a^{\prime}}\right)$ solution of the following minimization problem,

$$
\left\|w_{0}\right\|_{L^{2}\left(\partial D_{a^{\prime}}\right)}=\min _{\|K w-v\|_{\Xi \leq \delta}}\|w\|_{L^{2}\left(\partial D_{a^{\prime}}\right)} .
$$

Moreover, the classical theory implies that the solution $w_{0}$ of (3.34) 
belongs to $C\left(\partial D_{a^{\prime}}\right)$ and is the unique solution of

$$
\alpha_{0} w_{0}+K^{*} K w_{0}=K^{*} v \text {, for some } \alpha_{0} \text { such that }\left\|K w_{0}-v\right\|_{\Xi}=\delta \text {. }
$$

3.2. The general case $n \geq 2$. The proofs of Theorem 2.1, Corollary 2.2 as well as all the statements of the subsequent remarks offered above for the case $n=1$ can be immediately adapted to the general case $n \geq 2$ corresponding to two or more regions of control. The only major modifications are the fact that the space $\Xi$ is now defined by

$$
\Xi \equiv \prod_{i=0}^{n} L^{2}\left(\partial W_{n-i}\right),
$$

with the Hilbert topology induced by the usual scalar product and the fact that the operator $K: L^{2}\left(\partial D_{a^{\prime}}\right) \rightarrow \Xi$ is now given by

$$
K u\left(\mathbf{x}_{1}, \mathbf{x}_{2}, \ldots, \mathbf{x}_{n}, \mathbf{x}_{0}\right)=\left(K_{1} u\left(\mathbf{x}_{1}\right), K_{2} u\left(\mathbf{x}_{2}\right), \ldots, K_{n} u\left(\mathbf{x}_{n}\right), K_{0} u\left(\mathbf{x}_{0}\right)\right),
$$

for any $u \in L^{2}\left(\partial D_{a^{\prime}}\right)$, where

$$
K_{i} u\left(\mathbf{x}_{i}\right)=\int_{\partial D_{a^{\prime}}} u(\mathbf{y}) \frac{\partial \Phi\left(\mathbf{x}_{i}, \mathbf{y}\right)}{\partial \boldsymbol{\nu}_{\mathbf{y}}} d s_{\mathbf{y}}, \quad \text { for } \mathbf{x}_{i} \in \partial W_{i}, \quad \text { for } i=\overline{0, n} .
$$

4. Numerical support. In this section we will offer numerical support of the results presented in Theorem 2.1 in the context of the cloaking application as described in Remark 2.3. In what follows, $B_{r}(\mathbf{x})$ will denote the disk with radius $r$ and center $\mathbf{x} \in \mathbb{R}^{d}$.

In Figures 2 and 3 we plot the 2D $(d=2)$ results predicted by the above theory (see Remark 2.3) in the case $n=1, D_{a}=B_{0.1}(\mathbf{0}), W_{1}=$ $\{(r, \theta) \in(0.13,0.19) \times(2 \pi / 5,3 \pi / 5)\}, W_{0}=\mathbb{R}^{2} \backslash \bar{B}_{R}(\mathbf{0})$ with $R=10$, and the interrogating signal is a plane wave given by $u_{\text {int }}\left(x_{1}, x_{2}\right)=e^{-i k x_{2}}$, with wavenumber $k=10$. The plots in the top half of Figure 2 show the accuracy of the nulling obtained in the region of interest $W_{1}$, while the plots in the lower half of the figure show the necessary density $w_{j}$ for $\alpha_{j}=10^{-10}$ together with the field near $D_{a}$. We also remark that in fact the relative $L^{2}$ control error on $\partial W_{1}$ is $O\left(10^{-4}\right)$.

In Figure 3 we can see the small residual field existent on $\partial W_{0}$ as expected by the density result of Proposition 3.1 in the context of cloaking (see Remark 2.3). We used the Tikhonov regularization 

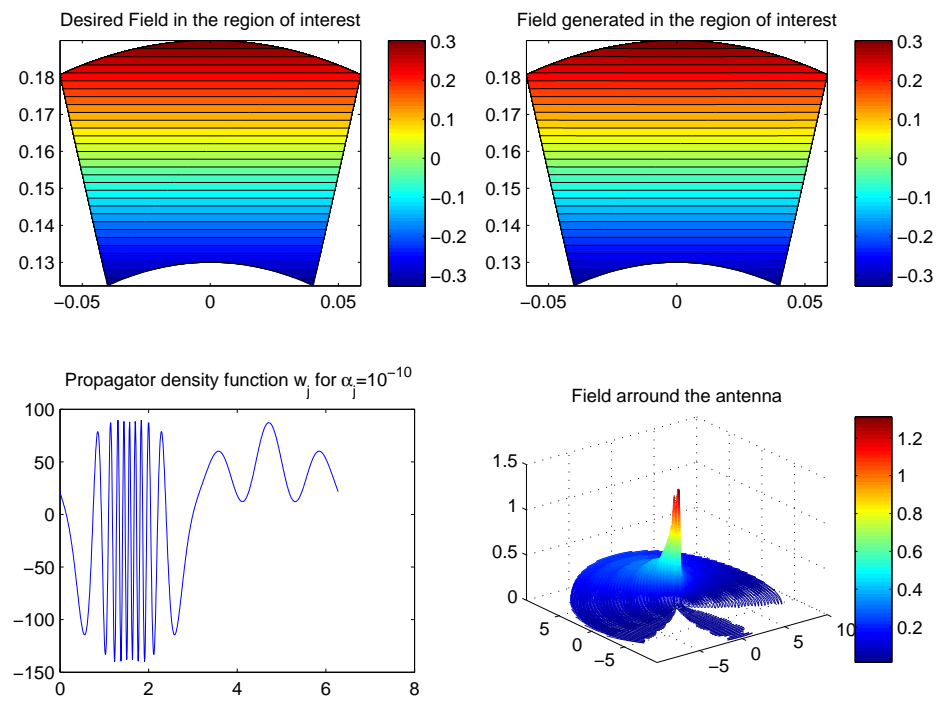

FIGURE 2. Numerical results in the two dimensions.

scheme to approximate the density function $w_{j}$ on several cross-sections around the center of the antenna (see Remark 3.2).

For 3D $(d=3)$ numerical support we consider $D_{a}(-l, l) \times B_{\delta}(\mathbf{0})$ and assume $W_{1}=\left\{B_{\delta+l_{1}+l_{2}}(\mathbf{0}) \backslash \bar{B}_{\delta+l_{1}}(\mathbf{0})\right\} \times(-p, p)$, where $l_{1}, l_{2}, p$ are given positive reals to be specified in the numerical tests. We also considered $W_{0}=(-L, L) \times B_{R}(\mathbf{0})$ for some $L>l$ and $R>l_{1}+l_{2}+\delta$.

In Figure 4, we sketch the geometry considered for the 3D numerical test. We will present the results predicted by the above theory (see Remark 2.3) with

$$
u_{i n t}(r, x)=J_{0}\left(\frac{r \chi_{1}}{R}\right) e^{i \beta x},
$$

where $J_{0}$ represents the 0 -th order Bessel- $J$ function, $\chi_{1}$ represents the first root of $J_{0}$, and $\beta=\sqrt{\left(k^{2}-\chi_{1}^{2} / R^{2}\right)}$.

We considered $k=6$ (corresponding to a frequency of $300 \mathrm{MHz}$ ), 


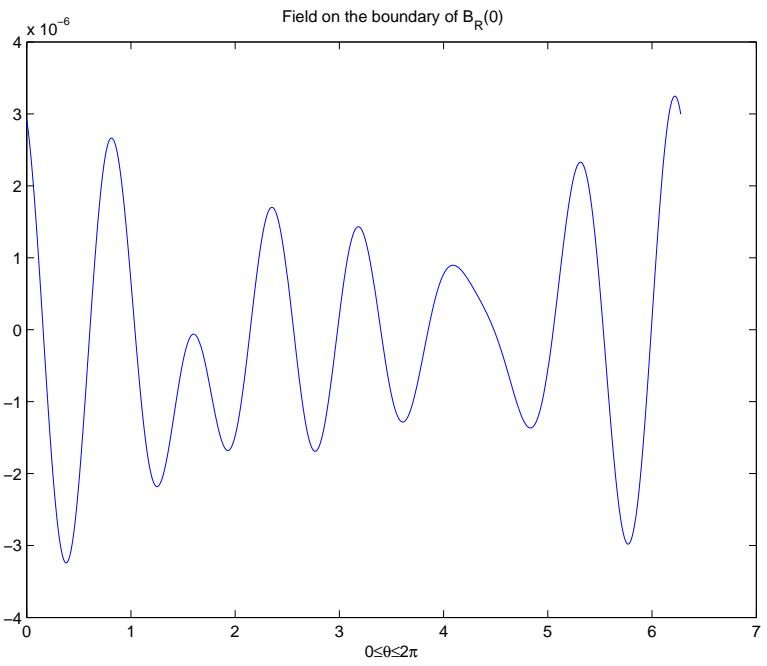

FiguRE 3. 2D accuracy of control on $\partial W_{0}=\partial B_{R}(\mathbf{0})$ with $R=10$

$\alpha_{j}=10^{-10}, \delta=0.01, l=0.3, l_{1}=0.1, l_{2}=0.04, p=0.1, R=15$ and $L=9$.

Figure 5 describes the contour plots of cross-sections of $-u_{\text {int }}$ (left column) versus the cross-sections of the field generated by the active antenna (right column). The three rows in Figure 5 represent the two fields in $W_{1}$ for three cross-section of the antenna, $x=-0.029$, $x=-0.0026, x=0.0237$, respectively.

Figures 6 and 7 each describe the density $w_{j}$ for $\alpha_{j}=10^{-10}$ for four cross sections along the negative and the positive $\mathrm{x}$-axis, respectively. Recall that $w_{j}$ introduced in (3.22) are functions of $(x, \theta)$ on $\partial D_{a}$. We used the Tikhonov regularization scheme to approximate the density function $w_{j}$ on several cross-sections around the center of the antenna (see Remark 3.2). The larger amplitudes towards the end of the 


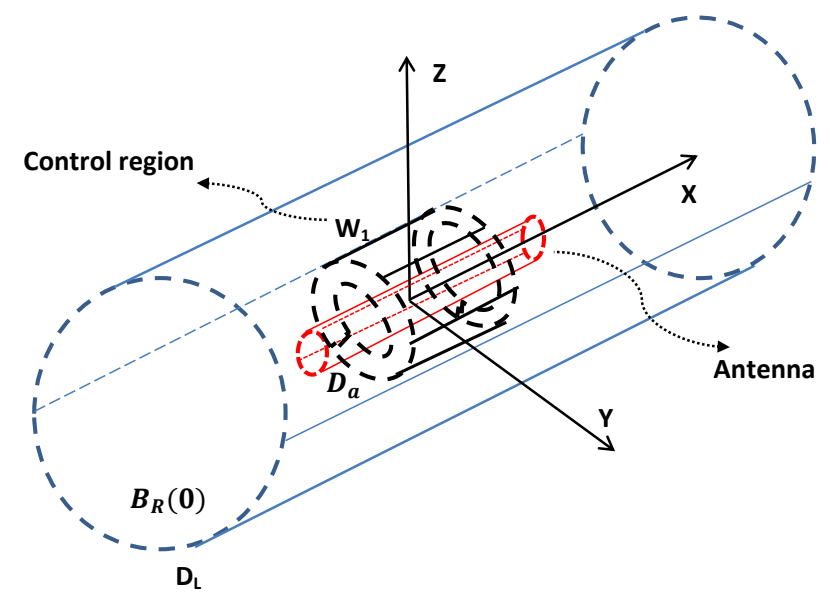

FigurE 4. Sketch of the geometry for the 3D numerics.

antenna are caused by the fact that $\partial D_{a}$ is not smooth in our numerical test. Even in this situation, one can see that the reasonable level of oscillations in $w_{j}$ as well as the small power needed when away from $\pm l$ are a strong indicator about the feasibility of such a scheme.

In Figure 8 we show two plots of the accuracy of the above 3D controls. The top plot shows the interpolation of 20 values of

$$
\frac{\left\|u_{j}(x, \cdot)+u_{i n t}(x, \cdot)\right\|_{L^{2}\left(\left\{B_{\delta+l_{1}+l_{2}}(\mathbf{0}) \backslash B_{\delta+l_{1}}(\mathbf{0})\right\} \times\{x\}\right)}}{\left\|u_{i n t}\right\|_{L^{2}\left(\partial W_{1}\right)}}
$$

for 20 linearly equally spaced values of $x$ in $[-0.1,0.1]$. The bottom plot in Figure 8 shows $\left\|u_{j}(x, \cdot)\right\|_{L^{2}\left(\partial B_{R}(\mathbf{0}) \times\{x\}\right)}$ for 200 linearly equally spaced values of $x$ in $[-9,9]$. Noting that on this particular cylindrical geometry it is enough to look at $L^{2}$ errors on cross sections along the main axis and that for $L$ large the value of $\left\|u_{j}( \pm L, \cdot)\right\|_{L^{2}\left(B_{R}(\mathbf{0})\right)}$ will be 

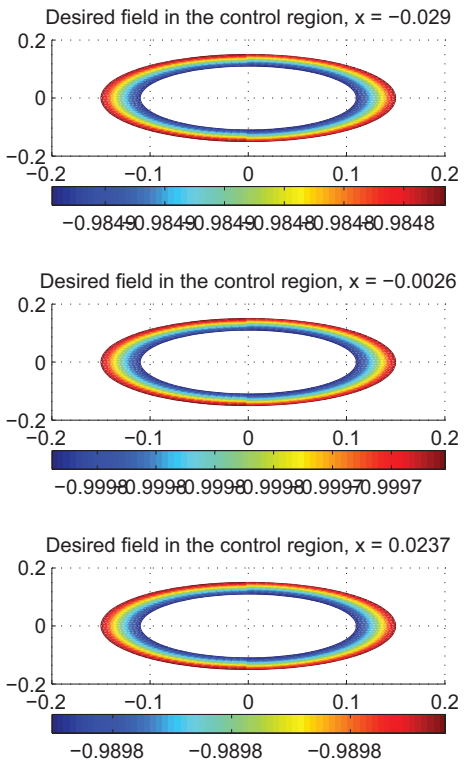
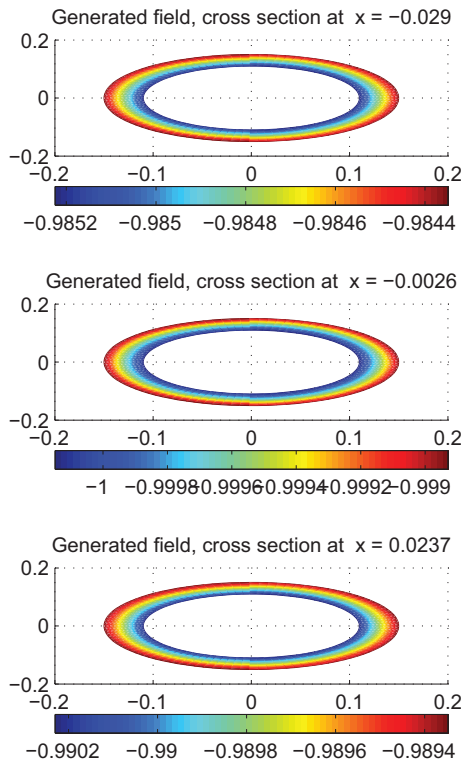

FiguRE 5. Accuracy of control in three different cross sections on the x-axis.

very small, we can observe the high accuracy of the control scheme.

5. A few remarks about stability. In this section we will offer a few insights into the important question of stability of the solution to problems (2.7) and (2.8). For simplicity, we consider in this section the case of cloaking as described in Remark 2.3 for $n=1$. Thus, let $\left(v_{0}, v_{1}\right)=\left(0,-u_{i n t}\right) \in \Xi$ where $u_{\text {int }}$ solves the Helmholtz equation in $B_{R}(\mathbf{0})$ and represents the interrogating field, and let $0<\delta \ll 1$ as above. Proposition 3.1 then implies that there exists a sequence of functions $w \in L^{2}\left(\partial D_{a^{\prime}}\right)$ such that

$$
\left\|K w+\left(0, u_{i n t}\right)\right\|_{\Xi} \leq \delta
$$



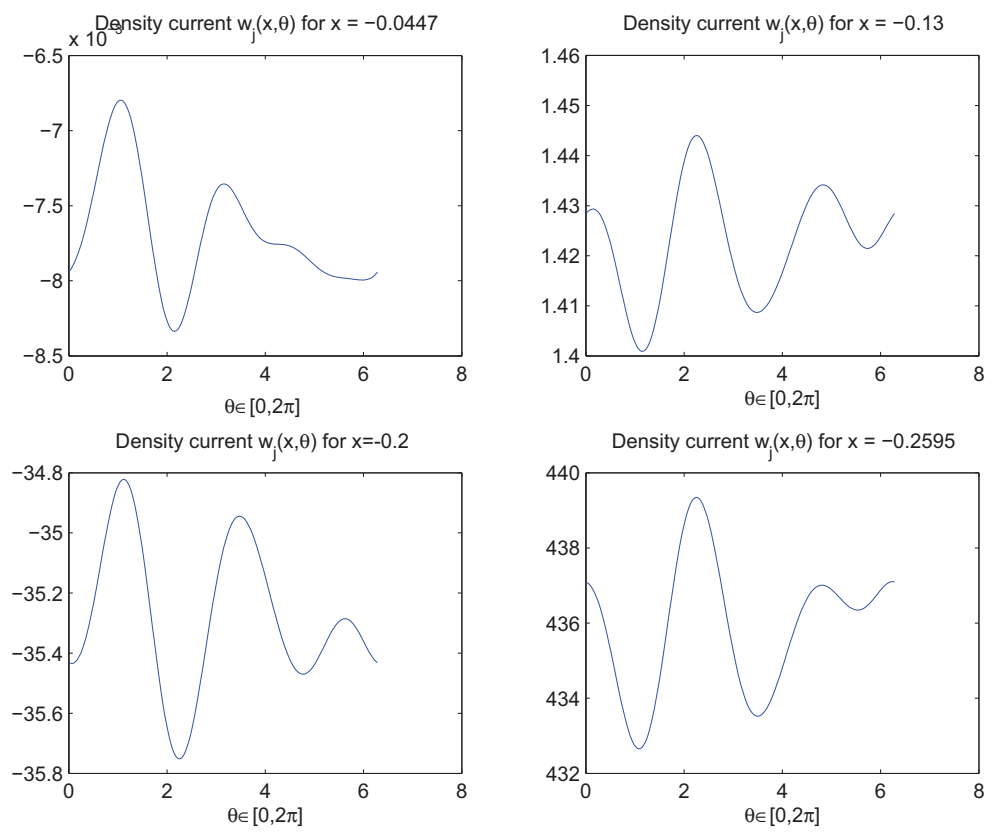

Figure 6. Density current $w_{j}(\theta)$ for four cross sections along the negative $x$ axis.

where $K$ was defined at (3.5) and the space $\Xi$ was introduced at (3.3). Remark 3.3 further provides the existence and characterization of a minimal energy solution for problem (5.1) in the sense of (3.34).

Note that, as shown at the end of the respective proof, the statement of Theorem 2.1 for $n=1$ is implied by (5.1) if one considers $v_{b}=\mathcal{D}_{1}(w)$ on $\partial D_{a}$, where the operator $\mathcal{D}_{1}$ was introduced in (3.6). Also observe that, by uniqueness, for $v_{b}=\mathcal{D}_{1}(w)$, problems (2.7) with (2.8) are equivalent to problem (5.1) above and thus can be viewed as a Fredholm equation of first kind associated to the compact operator $K$. It is well known that this type of problem is usually extremely ill-posed, and thus, the stability of the solution with respect to small perturbations 

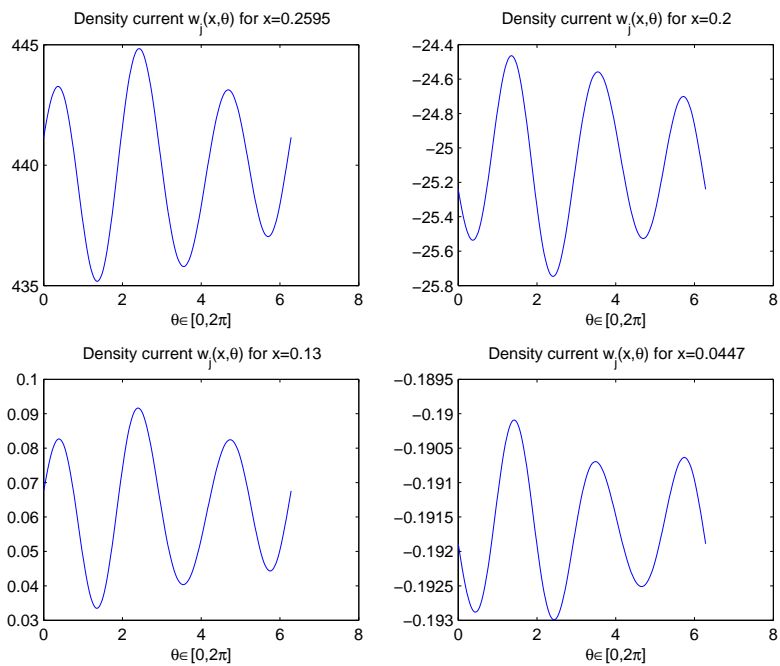

FIGURE 7 . Density current $w_{j}(\theta)$ for four cross sections along the positive $x$ axis

in $u_{\text {int }}$ is a major concern from the physical feasibility point of view. That is why we dedicated a separate research effort to this question. In this regard, in [20], we have completed a detailed sensitivity analysis of problem (5.1) where, in the context of cloaking and for several different types of interrogating fields, we studied the broadband character of the scheme, its stability and the power budget $\left(L^{2}\left(\partial D_{a}\right)\right.$-norm). Among other important observations, two major facts were strongly suggested by our sensitivity analysis:

A. The minimal norm solution for problem (5.1) will have a broadband character with very small $L^{2}\left(\partial D_{a}\right)$-norm and will be stable with respect to small perturbations in $u_{\text {int }}$, only in the case when $\operatorname{dist}\left(\partial W_{1}, \partial D_{a}\right)<<1$.

B. The case when $u_{\text {int }}$ corresponds to a plane wave appears more sensitive to measurement noise than the case when $u_{\text {int }}$ corre- 

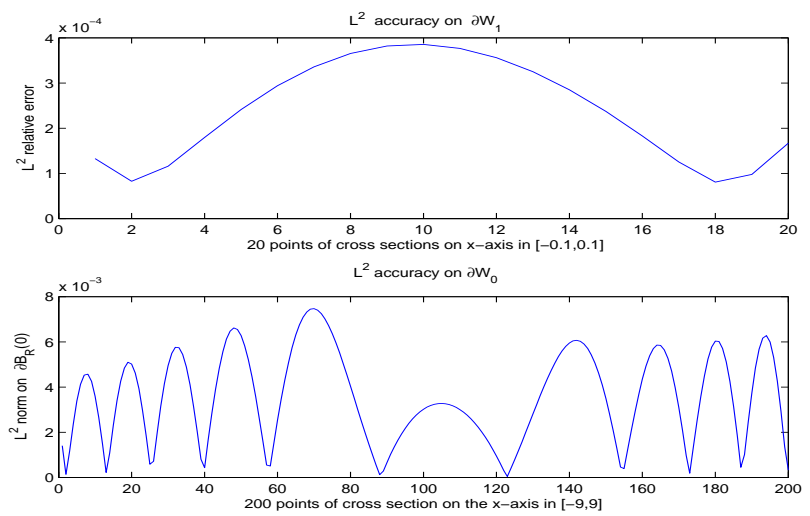

Figure 8. Control accuracy on $\partial W_{1}$ and $\partial W_{0}$.

sponds to a far field point source.

In what follows we only present two stability results for fixed frequency in 2D: one for $u_{\text {int }}(\mathbf{x})=\frac{i}{4} H_{0}^{(1)}\left(k\left|\mathbf{x}-\mathbf{x}_{0}\right|\right)$ with $\mathbf{x}_{0}=(-10000,0)$ and the other for $u_{i n t}\left(x_{1}, x_{2}\right)=e^{i k x_{1}}$. In both cases, we used Tikhonov regularization with the Morozov discrepancy principle for the choice of regularization parameter $\alpha$ to compute a minimal norm solution for the problem (5.1).

For the stability discussion, we considered a perturbed interrogating field $u_{i n t}^{\epsilon}$ with deterministic noise level of half percent, i.e.,

$$
\frac{\left\|u_{i n t}^{\epsilon}-u_{i n t}\right\|_{L^{2}\left(\partial W_{1}\right)}}{\left\|u_{i n t}\right\|_{L^{2}\left(\partial W_{1}\right)}}=\epsilon=0.005 .
$$

Then, we studied the power budget $\left(L^{2}\left(\partial D_{a}\right)\right.$-norm $)$ and stability of the minimal energy solution for the problem (5.1) as a function of $\mu=\operatorname{dist}\left(\partial W_{1}, \partial D_{a}\right)$. In each of the two cases introduced above, plane wave or point source interrogating signals, we respectively considered the power budget of the perturbed solution $\phi^{\epsilon}$ (i.e., minimal energy solution of (5.1) with $u_{i n t}^{\epsilon}$ as the interrogating field) and the relative $L^{2}$ error between $\phi^{\epsilon}$ and the unperturbed solution $\phi^{0}$ (i.e., minimal energy solution of (5.1) with $u_{\text {int }}$ as the interrogating field). 
In Figure 9 we assumed $n=1, D_{a}=B_{0.01}(\mathbf{0}), W_{1}=\{(r, \theta) \in(0.01+$ $\left.\mu, 0.014+\mu) \times\left(\frac{-\pi}{4}, \frac{\pi}{4}\right)\right\}$, for $\mu \in(0.001,0.03)$ and $W_{0}=\mathbb{R}^{2} \backslash \bar{B}_{R}(\mathbf{0})$ with $R=10$, wavenumber $k=10$, and the interrogating signal is a point source at $\mathbf{x}_{0}=(-10000,0)$ with $u_{\text {int }}\left(x_{1}, x_{2}\right)=\frac{i}{4} H_{0}^{(1)}\left(k\left|\mathbf{x}-\mathbf{x}_{0}\right|\right)$.

The left plot in Figure 9 shows the $L^{2}\left(\partial D_{a}\right)$-norm of the minimal energy solution $\phi^{\epsilon}$ for problem (5.1) with perturbed incident field $u_{\text {int }}^{\epsilon}$ defined as in (5.2) as a function of $\mu \in(0.001,0.03)$. The right plot in Figure 9 presents the $L^{2}\left(\partial D_{a}\right)$ relative difference between $\phi^{\epsilon}$ and $\phi^{0}$ and the minimal energy solutions of the perturbed and unperturbed problems, respectively, for $\mu \in(0.001,0.03)$. Note that $\left\|K \phi^{\epsilon}\right\|_{L^{\infty}\left(B_{R}(\mathbf{0})\right)} \approx O\left(10^{-5}\right)$.
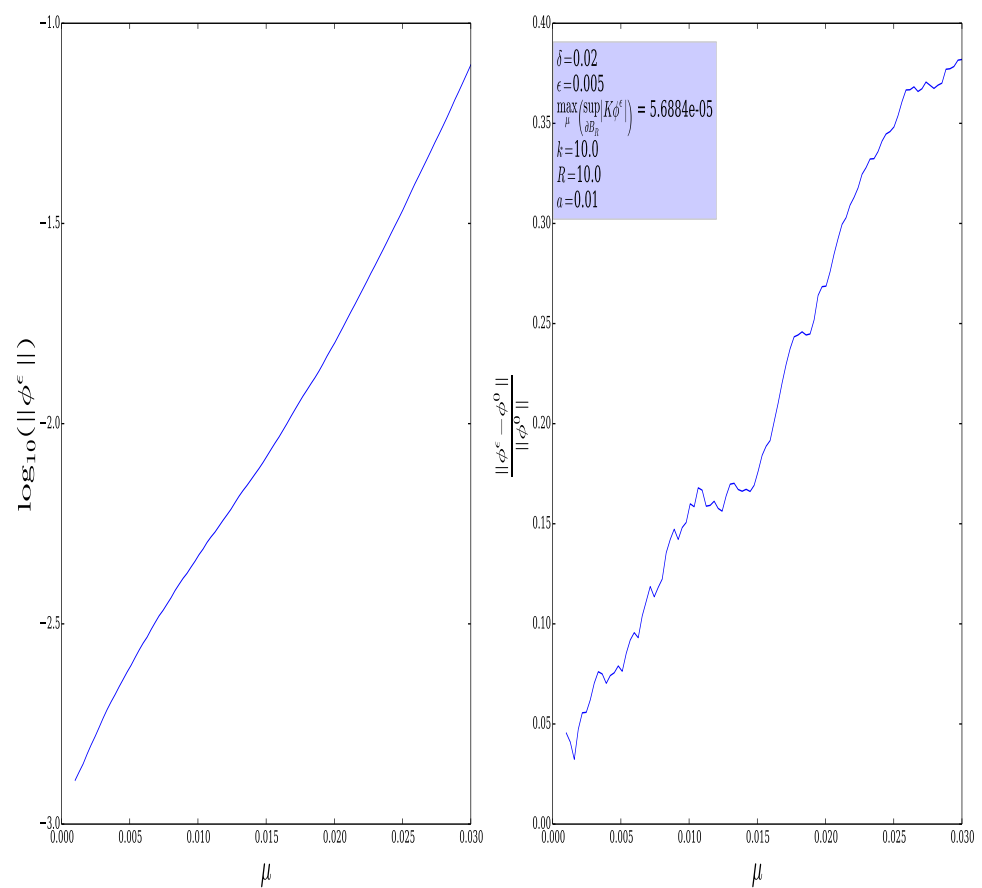

FIGURE 9. Stability for point source interrogating field as a function of $\mu=\operatorname{dist}\left(\partial W_{1}, \partial D_{a}\right)$. 
In Figure 10, we assumed $n=1, D_{a}=B_{0.01}(\mathbf{0}), W_{1}=\{(r, \theta) \in$ $(0.01+\mu, 0.014+\mu) \times(-\pi / 4, \pi / 4)\}$, for $\mu \in(0.001,0.03), W_{0}=$ $\mathbb{R}^{2} \backslash \bar{B}_{R}(\mathbf{0})$ with $R=200$, wavenumber $k=10$, and the interrogating signal is a plane wave given by $u_{i n t}\left(x_{1}, x_{2}\right)=e^{i k x_{1}}$.

The left plot in Figure 10 shows the $L^{2}\left(\partial D_{a}\right)$-norm of the minimal energy solution $\phi^{\epsilon}$ for the problem with perturbed incident field $u_{i n t}^{\epsilon}$ defined as in (5.2) as a function of $\mu \in(0.001,0.03)$. The right plot in Figure 10 describes the $L^{2}\left(\partial D_{a}\right)$ relative difference between $\phi^{\epsilon}$ and $\phi^{0}$ the minimal energy solutions of the perturbed and unperturbed problems, respectively, for $\mu \in(0.001,0.03)$. Observe that, in this case $\|\left. K \phi^{\epsilon}\right|_{L^{\infty}\left(B_{R}(\mathbf{0})\right)} \approx O\left(10^{-2}\right)$.
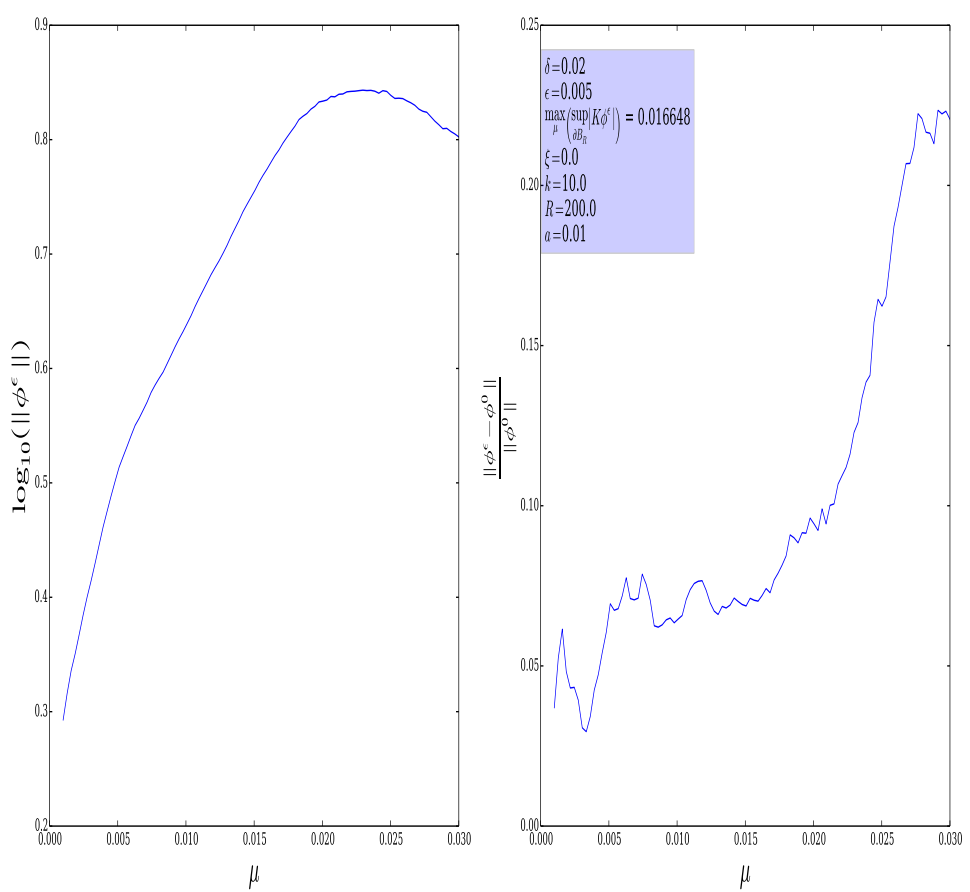

FIGURE 10. Stability for plane wave interrogating field as a function of $\mu=\operatorname{dist}\left(\partial W_{1}, \partial D_{a}\right)$. 
Comparing the two figures, one can observe that, even for $\mu<<1$ the $L^{2}\left(\partial D_{a}\right)$ norm of the solution is larger for the plane wave interrogating field than for the point source interrogating field. This is intuitive since, in the first case, one tries to approximate a plane wave with a decaying field, and this as expected will require a larger $L^{2}\left(\partial D_{a}\right)$ norm. Also, the decay of the solution is slower for the plane wave interrogating field than for the point source interrogating field, strengthening our conclusion that the former case is less feasible compared with the latter case.

In Figure 9, one can see the very small $L^{2}$ norm on $\partial D_{a}$ and relative good stability associated with $\operatorname{dist}\left(\partial W_{1}, \partial D_{a}\right)<<1$ for the case of an interrogating signal originating from a far field point source which are encouraging for the cloaking problem described in Remark 2.3.

The above numerical plots support the general sensitivity claims above for $k=10$ but a more complete discussion, analytic and numerical, is performed in [20]. We mention also that, for the above computations, we used a Fourier basis to represent the solution on $\partial D_{a}$. Hence, also encouraged by our current tests in the electromagnetic regime, we believe that these results can be dramatically improved if one considers a localized basis for the representation of the solution on $\partial D_{a}$, i.e., linear splines interpolation, and we plan to consider this type of basis for our future stability investigations.

6. Conclusions. We have theoretically shown the existence of a class of controls for the radiated solution of the scalar Helmholtz equation. Analytic results and numerical tests performed in [20] suggest that these solutions are stable, work over a broadband of frequencies and require realistic power budgets on the source only in near field regions, i.e., for control regions situated in the vicinity of the source. In this regard, in this paper we presented two finite frequency $2 \mathrm{D}$ numerical results.

We believe our results can be used for various applications where the control of acoustic fields in homogeneous isotropic media is of importance and we also perceive this paper as a first step towards understanding the more complex problem of control of electromagnetic fields and, in this regard in [1] and [37], we show, analytically and through real data simulations, that by cleverly using the theory of Debye potentials (which provide the link between scalar Helmholtz fields 
and electromagnetic fields) one can prove similar results in the context of near field control of normal modes in waveguide and of free space electromagnetic fields. In our work, we build on the single antenna case and propose active arrays to control acoustic or electromagnetic fields in the near field regions.

Although equally important for the practical point of view, there are important technical differences between the acoustic regime where, through transducers, a speed differential can be obtained but the environment may be heterogeneous, and the electromagnetic regime where there is no speed differential but the environment is homogeneous. Also, defending a fixed site (e.g., ground building) where the communication with the exterior may be maintained through underground wires, is less challenging than defending a moving airplane, for example, where the doppler effect must be considered and where maintaining communication capabilities and differentiating between friendly and enemy signals are additional challenges.

Finally, we mention that in any real life application of such results, one either needs to know the nature (phase, and magnitude) of the interrogating field or one needs to pair the finite frequency (i.e., timeharmonic) scheme with a feedback time-control loop for the sensing and countering of unknown interrogating signals.

Although our current results, including the forthcoming works $[\mathbf{1}, \mathbf{3 7}]$, consider only time-harmonic fields with a priori known interrogating signals, as a next step we are planning to consider the full time-domain problem first for a case of a dipole antenna defending a conducting surface and then subsequently for planar and, respectively, conformal antenna arrays defending a given site.

Acknowledgments. The author would like to thank Dr. Richard Albanese and Dr. Richard Medina from ADED LLC for stimulating discussions and for encouraging this work. The author is also grateful to Dr. Mark Hubenthal for his help with the final presentation of this work.

\section{REFERENCES}

1. R. Albanese and D. Onofrei, Near field control of transverse modes in open waveguides, submitted. 
2. A. Alu and N. Engheta, Plasmonic and metamaterial cloaking: Physical mechanism and potentials, Pure Appl. Opt. 10 (2008).

3. A. Bakushinsky and A. Goncharsky, Ill-posed problems: Theory and applications, Kluwer Academic Publishers, Denmark, 1994.

4. H. Chen and C. Chan, Acoustic cloaking and transformation acoustics, J. Appl. Phys. 43 (2010).

5. H. Chen, C.T. Chan and P. Sheng, Transformation optics and metamaterials, Nature Mat. 9 (2010), 387-396.

6. D. Colton and R. Kress, Inverse acoustic and electromagnetic scattering theory, Springer-Verlag, New York, 1998.

7. _ Integral equation methods in scattering theory, Pure Appl. Math., Wiley Interscience, New York, 1983.

8. S.A. Cummer, B.-I. Popa, D. Schurig, D.R. Smith, J. Pendry, M. Rahm and A. Starr, Scattering theory derivation of a 3d acoustic cloaking shell, Phys. Rev. Lett. 100 (2008).

9. J. Du, S. Liu and Z. Lin, Broadband optical cloak and illusion created by the low order active sources, Opt Express. 20 (2012), 8608-8617.

10. H.W. Engl, M. Hanke and A. Neubauer, Regularization of inverse problems, Math. Appl. 175, Kluwer Academic Publishers, Springer, New York, 1996.

11. S.J. Elliot and P.A. Nelson, The active control of sound, Electr. Comm. Eng. J., August, 1990.

12. C.R. Fuller and A.H. von Flotow, Active control of sound and vibration, IEEE, 1995.

13. A. Greenleaf, Y. Kurylev, M. Lassas and G. Uhlmann, Invisibility and inverse problems, Bull. Amer. Math. Soc. 46 (2009), 55-97.

14. A. Greenleaf, M. Lassas and G. Uhlmann, Anisotropic conductivities that cannot be detected by eit, Phys. Meas. 24 (2003), 413-419.

15. , On nonuniqueness for Calderón's inverse problem, Math. Res. Lett. 10 (2003), 685-693.

16. F. Guevara Vasquez, G.W. Milton and D. Onofrei, Active exterior cloaking, Phys. Rev. Lett. 103 (2009).

17. F. Guevara Vasquez, G.W. Milton, D. Onofrei and P. Seppecher, Transformation elastodynamics and active exterior acoustic cloaking, Acoustic metamaterials: Negative refraction, imaging, lensing and cloaking, Springer Ser. Mat. Sci. 166 (2013), 289-318.

18. Broadband exterior cloaking, Optics Expr. 17 (2009), 14800-14805.

19. Exterior cloaking with active sources in two dimensional acoustics, Wave Motion 48 (2011), 515-524.

20. M. Hubenthal and D. Onofrei, Qualitative comparison between near field vs far field control of radiated fields modeled by Helmholtz equation, submitted.

21. O.D. Kellogg, Foundations of potential theory, Dover Books on Physics, New York, 2010. 
22. R. Kress, Linear integral equations, Appl. Math. Sci., Springer-Verlag, New York, 1999.

23. Y. Lai, H. Chen, Z.-Q. Zhang and C.T. Chan, Complementary media invisibility cloak that cloaks objects at a distance outside the cloaking shell, Phys. Rev. Lett. 102 (2009).

24. U. Leonhardt, Notes on conformal invisibility devices, New J. Phys. 8 (2006), 118.

25. Optical conformal mapping, Science 312 (2006), 1777-1780.

26. P. Leug, Process of silencing sound oscillations, U.S. patent no. 2043416, 1936.

27. J. Loncaric, V.S. Ryaben'kii and S.V. Tsynkov, Active shielding and control of environmental noise, technical report, NASA/CR-2000-209862, ICASE Report No. 2000-9, 2000.

28. J. Loncaric and S.V. Tsynkov, Quadratic optimization in the problems of active control of sound, Appl. Numer. Math. 52 (2005), 381-400.

29. Q. Ma, Z.L. Mei, S.K. Zhu and T.Y. Jin, Experiments on active cloaking and illusion for Laplace equation, Phys. Rev. Lett. 111 (2013).

30. D.A.B. Miller, On perfect cloaking, Opt. Express 14 (2006), 12457-12466.

31. G.W. Milton and N.-A.P. Nicorovici, On the cloaking effects associated with anomalous localized resonance, Proc. Roy. Soc. Lond. Math. Phys. Sci. 462 (2006), 3027-3059.

32. G.W. Milton, N.-A.P. Nicorovici, R.C. McPhedran, K. Cherednichenko and Z. Jacob, Solutions in folded geometries, and associated cloaking due to anomalous resonance, New J. Phys. 10 (2008).

33. N.-A.P. Nicorovici, G. Milton, R.C. McPhedran and L.C. Botten, Quasistatic cloaking of two-dimensional polarizable discrete systems by anomalous resonance, Opt. Express 15 (2007), 6314-6323.

34. A.N. Norris, F.A. Amirkulova and W.J. Parnell, Source amplitudes for active exterior cloaking, Inverse Prob. 28 (2012).

35. H.F. Olson and E.G. May, Electronic sound absorber, J. Acad. Soc. Amer. 25 (1953), 1130-1136.

36. D. Onofrei, On the active manipulation of fields and applications, I - The quasistatic regime, Inv. Prob. 28 (2012).

37. _ Near field control of free space electromagnetic fields, in preparation.

38. N. Peake and D.G. Crighton, Active control of sound, Annu. Rev. Fluid Mech. 32 (2000), 137-164.

39. J.B. Pendry, D. Schurig and D.R. Smith, Controlling electromagnetic fields, Sci. 312 (2006), 1780-1782.

40. A.W. Peterson and S.V. Tsynkov, Active control of sound for composite regions, SIAM J. Appl. Math. 67 (2007), 1582-1609.

41. M. Selvanayagam and G.V. Eleftheriades, An active electromagnetic cloak using the equivalence principle, IEEE Ant. Wireless Prop. Lett. 11 (2012). 
42. M. Selvanayagam and G.V. Eleftheriades, Experimental demonstration of active electromaagnetic cloaking, Phys. Rev. 3 (2013).

43. H.H. Zheng, J.J. Xiao, Y. Lai and C.T. Chan Exterior optical cloaking and illusions by using active sources: A boundary element perspective, Phys. Rev. 81 (2010).

Department of Mathematics, University of Houston, Houston, TX 77004 Email address: onofrei@math.uh.edu 